\title{
A Method for Multimedia User Interface Design for Mobile Learning
}

\author{
Authors : Shimaa Nagro, Russell Campion
}

Abstract : Mobile devices are becoming ever more widely available, with growing functionality, and are increasingly used as an enabling technology to give students access to educational material anytime and anywhere. However, the design of educational material user interfaces for mobile devices is beset by many unresolved research issues such as those arising from emphasising the information concepts then mapping this information to appropriate media (modelling information then mapping media effectively). This report describes a multimedia user interface design method for mobile learning. The method covers specification of user requirements and information architecture, media selection to represent the information content, design for directing attention to important information, and interaction design to enhance user engagement based on HumanComputer Interaction design strategies (HCI). The method will be evaluated by three different case studies to prove the method is suitable for application to different areas / applications, these are; an application to teach about major computer networking concepts, an application to deliver a history-based topic; (after these case studies have been completed, the method will be revised to remove deficiencies and then used to develop a third case study), an application to teach mathematical principles. At this point, the method will again be revised into its final format. A usability evaluation will be carried out to measure the usefulness and effectiveness of the method. The investigation will combine qualitative and quantitative methods, including interviews and questionnaires for data collection and three case studies for validating the MDMLM method. The researcher has successfully produced the method at this point which is now under validation and testing procedures. From this point forward in the report, the researcher will refer to the method using the MDMLM abbreviation which means Multimedia Design Mobile Learning Method.

Keywords : human-computer interaction, interface design, mobile learning, education

Conference Title : ICMLOL 2017 : 19th International Conference on Mobile Learning and Online Learning

Conference Location : Dubai, UAE

Conference Dates : February 26-27, 2017 